\title{
Noise simulation and low noise design of skinned panel structure of scientific experiment rack
}

\author{
Changshuai $\mathrm{Yu}^{1,2,3^{*}}$, Zhong Luo,3, Haitao Luo ${ }^{1,2}$, Siwei $\mathrm{Guo}^{3}$, Guangming Liu ${ }^{1,2}$, and \\ Zhenxin $\mathrm{Li}^{1,2}$ \\ ${ }^{1}$ Key Laboratory of Robotics, Shenyang Institute of Automation, Chinese Academy of Sciences, \\ Shenyang 110016, China \\ ${ }^{2}$ Institutes for Robotics and Intelligent Manufacturing, Chinese Academy of Sciences, Shenyang \\ 110169, China \\ ${ }^{3}$ School of Mechanical Engineering and Automation, Northeastern University, Shenyang Liaoning \\ 110819, China.
}

\begin{abstract}
The noise of the scientific experiment rack radiates into the space station through the skinned structure, which directly affects the safety and health of astronauts in orbit for a long time, so it is necessary to carry out low-noise design. Firstly, the finite element model of the panel structure is established, and the correctness of the model is verified by modal test. Secondly, select a point as the vibration excitation point on the finite element model of the plate structure to simulate the vibration input of the excitation source, obtain its vibration response through the modal superposition method, take the vibration response as the boundary condition of the acoustic boundary element, use the modal acoustic transfer vector technology to calculate the radiation noise of the plate structure, and verify it through the noise test in the half anechoic chamber. Then, the acoustic pressure contribution analysis of the radiated noise from the skinned panel structure is carried out, and the panel area which can reduce the radiated noise of the target is determined. The constrained damping layer is applied in this area. The results show that the radiated noise at the target position is significantly reduced.
\end{abstract}

\section{Introduction}

Noise is one of the important environmental factors of manned spacecraft. Increased environmental noise of spacecraft will directly affect the communication between astronauts, interfere with astronauts' sleep, and disturb the attention of astronauts, which may affect space missions ${ }^{[1]}$. The International Space Station currently operating in orbit has many problems with excessive noise. For example, some airlock decompression pumps in the United States, some centrifuge rotors in Japan, and some European microgravity scientific glove box systems have far higher noise levels than the standard requirements ${ }^{[2-3]}$. Therefore, the implementation of noise control during the design process of space station is the most

\footnotetext{
*Corresponding author: yuchangshuai@sia.cn
} 
economical. The vibration and noise reduction index of the sub-system equipment, the design of the ground test plan and the test conditions should be proposed as soon as possible. Meanwhile, the accurate sound and vibration environment prediction is basis to guide the design of noise control of the space station system ${ }^{[4]}$.

The methods of low-frequency noise prediction in space stations are commonly used units discrete methods of acoustic finite element and acoustic boundary element ${ }^{[5]}$. Larbi $\mathrm{W}^{[6]}$ proposed a coupled finite element/boundary element method (FEM/BEM) simulation method considering the piezoelectric dampers on the structure surface; Citarella $\mathrm{R}^{[7]}$ uses the structural finite element/acoustic boundary element coupling method to simulate the lowfrequency radiation noise of the car, which is compared with the structural finite element/acoustic finite element coupling method and experimental data.

Based on the structural finite element/acoustic boundary element coupling and modal acoustic transfer vector technology, this paper calculates the low-frequency radiation noise of a skinned panel structure of Scientific Experiment Rack, and conducts experimental verification; Firstly, divide the panel structure into different areas and analyze the acoustic contribution of different areas of the panel surface on the astronaut's noise in order to determine the area that contributes positively to the radiation noise. Then, apply a constrained damping layer on this area to reduce the vibration, which can achieve the purpose of the lownoise radiation design of the scientific experiment rack.

\section{Establishment of panel structure model}

Our country is constructing space station and carrying out space science experiments by using space microgravity environment is an important part of the space station. There are many scientific experiment rack systems in the cabin for scientific experiments ${ }^{[11]}$, as shown in Figure. 1. The experiment rack is mainly used for scientific experiments by astronauts. The main function of the environmental control system of the experiment rack is to maintain the temperature and humidity of the experiment rack, which is one of the main vibration sources of the experimental racks and finally radiates noise through the experiment rack skinned panel structure.

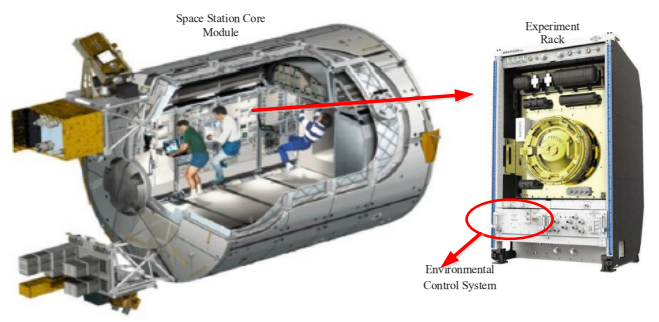

Fig. 1. Space station internal structure and scientific experiment rack.

\subsection{Establishment of finite element model of panel structure}

The research object of this paper is the skinned panel structure of the environmental control system of the space station scientific experiment rack. The main function of the environmental control system is to maintain the temperature and humidity inside the experimental cabinet. Therefore, the skinned panel structure usually carries vibration sources such as fans and motors. The vibration sources are transmitted to the panel through vibration. Then the panel radiates noise.

The simulation part of the skinned panel structure of the environmental control system has the characteristics of light weight and strong bearing capacity whose structural material 
is aluminum, and the surface of the skin is designed with a middle rib structure, as shown in the figure. 2.

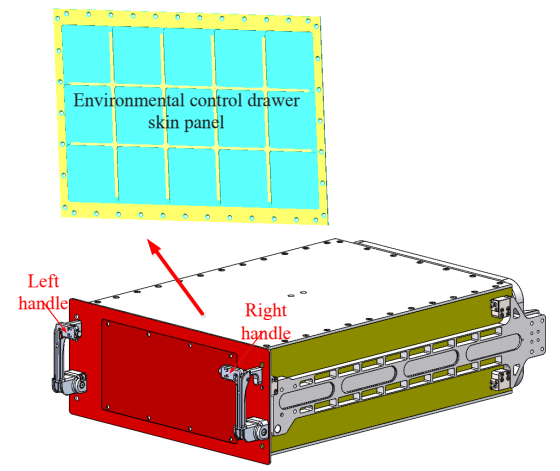

Fig. 2. Panel structure of experimental rack.

After establishing the finite element model, solve the modal of the structure in the free state. The first 6-order modal results of the panel structure are shown in Figure .3 .

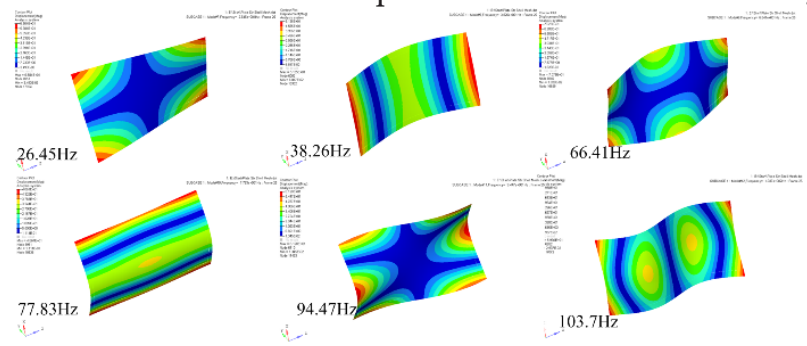

Fig. 3. Modal results of panel.

\subsection{Acoustic boundary element model}

In order to study the noise radiation of the skinned panel structure, the acoustic simulation analysis adopts the method of acoustic boundary element. Firstly, based on the finite element model of the panel structure, the acoustic boundary element mesh is constructed. The modal acoustic transfer vector technology is used to calculate the radiation noise of the panel structure, which can greatly improve the calculation efficiency. At the same time, the symmetry plane technology is used to simulate the impact of the reflection of the floor of the experimental rack on the sound propagation. The simulation model of the panel structure is shown in the figure. 4.

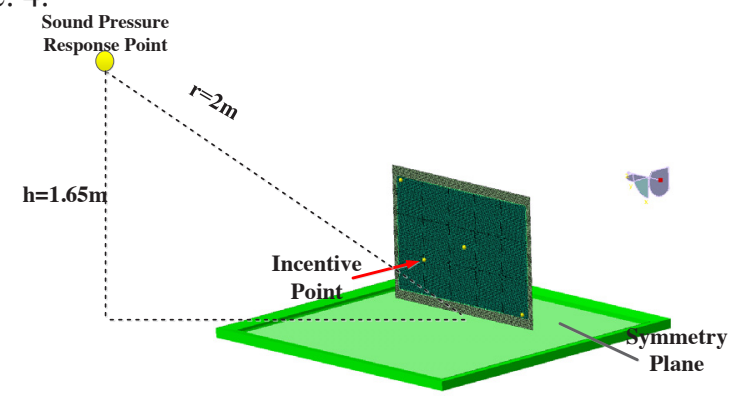

Fig. 4. Acoustic simulation model of plate structure. 
Input the force spectrum at the excitation point to simulate the input of the fan, perform sound pressure simulation for sound pressure response points. The position of sound pressure response point selected to simulate the position of the astronaut's ears is 2 meters away from the center of the symmetry plane and the vertical distance from the symmetry plane is $1.65 \mathrm{~m}$.

\section{Structural vibration and noise simulation and experimental verification}

\subsection{Acoustic transfer vector ATV}

This paper analyzes the radiation noise of the skin panel based on the acoustic transfer vector, which expresses the relationship between the vibration velocity input of the radiating surface of the structure and the sound pressure of the field point, as shown in the following formula:

$$
[p(\omega)]=\{\operatorname{ATV}(\omega)\}^{\mathrm{T}}\left\{v_{n}(\omega)\right\}
$$

where $\omega$ is the angular frequency; $\{\mathrm{ATV}\}$ is the sound transfer vector; $\left\{v_{n}\right\}$ is the vibration on the radiating surface of the structure. The sound transfer vector can be interpreted as a collection of sound transfer functions from a surface node to a single field point, which only depends on the configuration of the acoustic domain, such as the geometric structure and characteristics (sound velocity and density), acoustic surface treatment, frequency and field point, which do not rely on load conditions and structural response. Modal acoustic transfer vector is a more effective acoustic response calculation method compared with the acoustic transfer vector. It is not necessary to input the surface velocity of the skin panel $\left[v_{n}\right]$, introduce the modal feature vector $\left[\phi_{n}\right]$ and related modal coordinates $\left[\xi_{\omega}\right]$, as shown in the following formula:

$$
\left\{v_{n}(\omega)\right\}=j \omega\left[\phi_{n}\right]\{\xi(\omega)\}
$$

Combining formula (1), we can get

$$
[p(\omega)]=[\operatorname{MATV}(\omega)]\{\xi(\omega)\}
$$

In the formula: $[\operatorname{MATV}(\omega)]$ is the modal acoustic transfer vector, as shown in the following formula:

$$
[\operatorname{MATV}(\omega)]=j \omega[\operatorname{ATM}(\omega)]\left[\phi_{n}\right]
$$

\subsection{Vibration and noise simulation}

As shown in the acoustic simulation model of the plate structure in Figure 7, the force spectrum is input at the position of the excitation point, and the input waveform of the force spectrum is shown in the figure .8 .
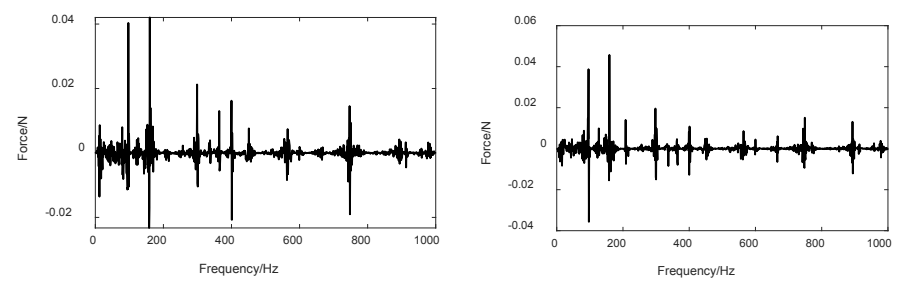

(1)Real part (2)Imaginary part

Fig. 8. Incentive input. 
After simulation by method of modal acoustic transfer vector, the output response of the sound pressure response point is shown in the figure. 5 .

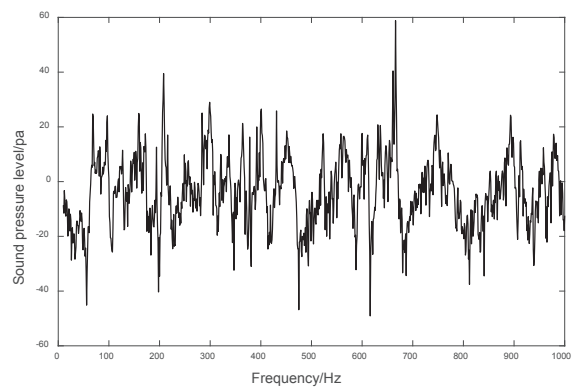

Fig. 5. Response curve of sound pressure response point.

It can be seen from Fig. 10 that the sound pressure response points have peaks at $97 \mathrm{~Hz}$, $159 \mathrm{~Hz}, 208 \mathrm{~Hz}, 299 \mathrm{~Hz}, 401 \mathrm{~Hz}, 666 \mathrm{~Hz}, 748 \mathrm{~Hz}$, and $893 \mathrm{~Hz}$.

\subsection{Test verification}

The above simulation results will be tested and verified next. In order to prevent the influence of echo on the test results, the test was carried out in a half anechoic chamber, where the position of excitation point, acceleration response point and sound pressure response point were consistent with the simulation. The symmetry plane in the ground simulation of the half anechoic chamber is shown in the figure. 6 . The skinned panel is suspended with elastic ropes to simulate the free boundary condition, and the vibration exciter is used to input the force spectrum at the excitation point, which is consistent with the simulation.

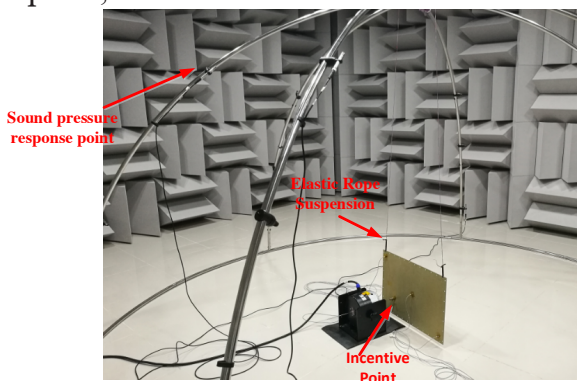

Fig. 6. Plate structure test verification.

The comparison between the test and the simulation of the sound pressure response point is shown in the figure 7.

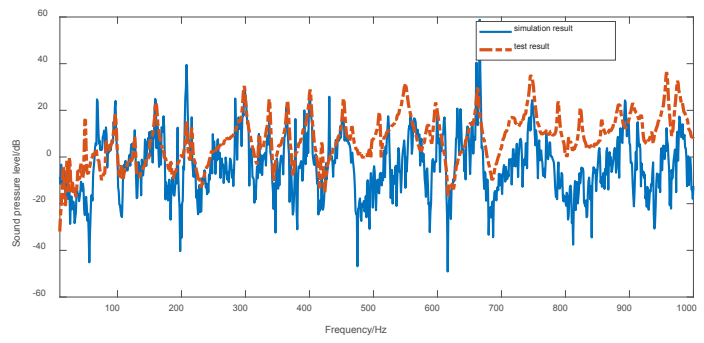

Fig. 7. Sound pressure comparison of test and simulation. 


\section{Low noise design of panel structure of scientific experiment rack}

\subsection{Analysis of sound pressure contribution}

The plate structure of the experiment rack is divided into different areas, as shown in the figure. 8. The noise felt by the astronaut is generated by the superposition of the sound pressure components caused by panels at the positions of the astronaut's ears. Different areas of the panel have different acoustic contributions to the position of the astronaut's ears, and are generally divided into positive contribution areas, negative contribution areas and neutral contribution areas. The sound pressure response at the position of the astronaut's ear increases with the growth of the vibration velocity in the positive contribution area, and decreases with the growth of the vibration velocity in the negative contribution area. Therefore, in order to improve the noise environment of astronauts in orbit, it is necessary to analyze the acoustic contribution of the panels and determine the effective modification area.

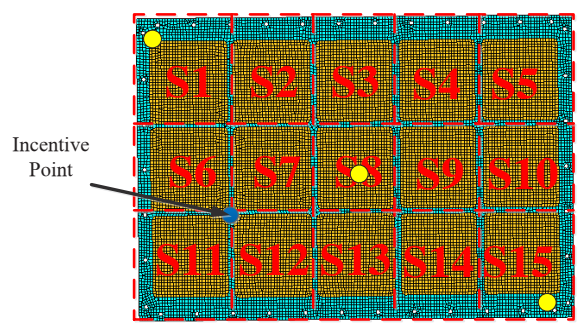

Fig. 8. Division of plate structure area.

Based on the assumption of linear acoustics, the complex sound pressure at the astronaut's ear is the superposition of the complex sound pressure response transmitted from the vibration of each unit on the structural plane of the thermal control drawer plate to that point, which is formula (4). Considering the problem of vibration and noise at the frequency point of $\omega$, the projection of the complex sound pressure caused by the vibration of a certain element in the $\mathrm{S}$ plane at the field point to the total sound pressure of the field point is called the sound pressure contribution De of the element on the S plane, and the formula is:

$$
D_{e}(\omega)=\operatorname{Re} \frac{p_{e}(\omega) \bar{p}(\omega)}{|p(\omega)|}
$$

where $p(\omega)$ is the total complex sound pressure at the astronaut's ear, $p_{e}(\omega)$ is the complex sound pressure caused by a unit on the $\mathrm{S}$ surface, $\mathrm{Re}$ is the complex real part operator. Divide the $\mathrm{S}$ plane into 15 regions, as shown in the figure. 15. Through the superposition of units, the sound pressure caused by all the units on the $S_{i}(i=1,2 \cdots 15)$ is:

$$
P_{C}(\omega)=\sum_{m_{i}} P_{e}(\omega)
$$

where $m_{i}$ is the number of elements in the $S_{i}(i=1,2 \cdots 15)$.

Therefore, the contribution of the $S_{i}(i=1,2 \cdots 15)$ at a certain frequency is:

$$
D_{C}(\omega)=\operatorname{Re} \frac{p_{C}(\omega) \bar{p}(\omega)}{|p(\omega)|}
$$

It can be seen from the definition of the above formula that there are positive contributions and negative contributions. The positive contribution indicates that the vibration of the panel is positively related to the total sound pressure, while the negative contribution is the opposite. 
The positive contribution must be increased to partially suppress the noise. Select the three largest frequency peaks of the sound pressure response curve, namely $666 \mathrm{~Hz}, 208 \mathrm{~Hz}, 299 \mathrm{~Hz}$ to analyze the sound pressure contribution of response points. The sound pressure contribution of different areas of the plate structure at the three frequencies of $666 \mathrm{~Hz}, 208 \mathrm{~Hz}$, $299 \mathrm{~Hz}$ are shown as follows.
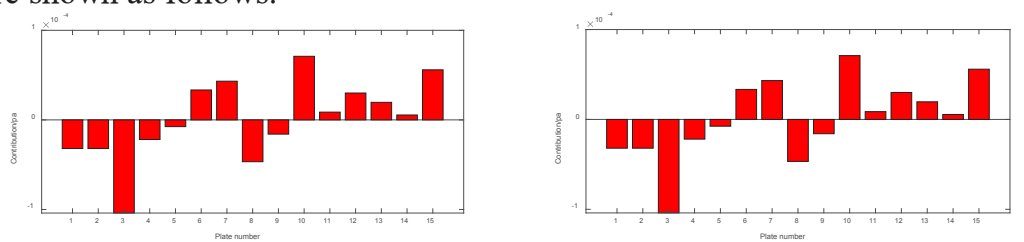

(1) $666 \mathrm{~Hz}$ contribution of sound pressure (2) $208 \mathrm{~Hz}$ contribution of sound pressure

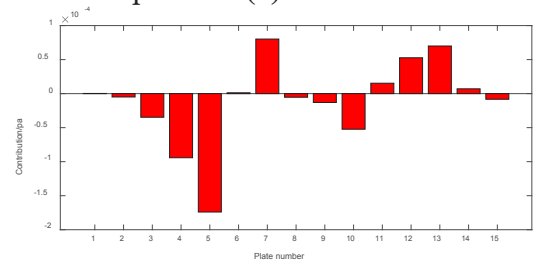

(3) $299 \mathrm{~Hz}$ contribution of sound pressure

Fig. 9. Contribution of sound pressure.

As can be seen from the figure. 9, areas 6, 7, 12, 13 play a positive contribution on the sound pressure of the three frequencies at the same time.

\subsection{Constrained damping layer to reduce vibration and noise}

This article applies constrained damping layer treatment to areas numbered 6, 7, 12, and 13 (as shown in the figure. 10). The thickness of the damping layer is $3 \mathrm{~mm}$. The parameters of the damping layer are: shear modulus is $1.5 \mathrm{Mpa}$, Poisson's ratio is 0.45 , the density is $10 \mathrm{~g} / \mathrm{m} 3$, the loss factor is 1.25; the thickness of the constraining layer is $1 \mathrm{~mm}$, and the material is aluminum.

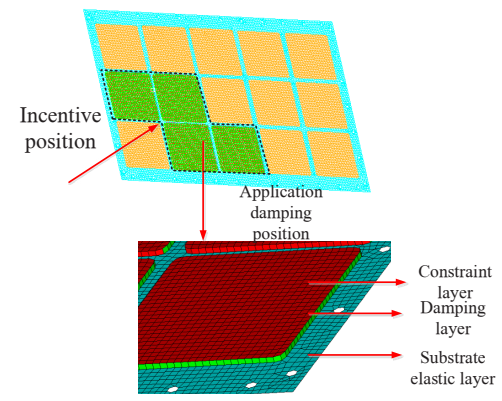

Fig. 10. Position of constrained damping layer.

After simulation analysis, the comparison of the sound pressure level of response points between the coated and uncoated constrained damping layer is as shown in Fig. 11. 


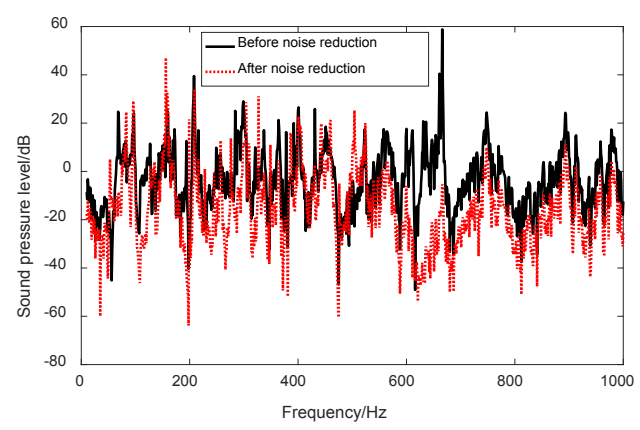

Fig. 11. Comparison of sound pressure levels before and after noise reduction.

It can be seen that sound pressure level among multiple frequency bands are significantly reduced. After $600 \mathrm{~Hz}$, the sound pressure value after noise reduction is significantly lower than that before noise reduction. The sound pressure peak at $666 \mathrm{~Hz}$ is significantly smaller, the noise reduction amplitude reaches $64 \mathrm{~dB}$, the noise reduction amplitude at $208 \mathrm{~Hz}$ is $20 \mathrm{~dB}$, noise reduction amplitude at $299 \mathrm{~Hz}$ is $0.3 \mathrm{~dB}$. Although the sound pressure peak at individual frequency points becomes larger, the overall acoustic environment at the sound pressure response point is significantly improved.

\section{Conclusion}

This paper takes the skinned panel structure of the space station experiment rack as the research object, uses the finite element method to simulate the structure dynamics of the skin panel. The panel vibration response obtained by the finite element simulation is used as the boundary condition of the acoustic boundary element, and the modal acoustic transfer vector technology is used to calculate the low-frequency radiation noise of the panel structure. The noise test in the half anechoic chamber verifies the correctness of the noise simulation method. The sound pressure contribution of the radiated noise of the skinned panel structure is analyzed, the noise reduction area is determined and constrained damping is performed to reduce the noise. The result shows that the radiated noise at the target position is significantly reduced.

The author(s) disclosed receipt of the following financial support for the research, authorship, and/or publication of this article: this research was financially supported by Liao Ning Revitalization Talents Program (XLYC1907152); Natural Science Foundation of Liaoning Province (2020-MS-029 and 2019MS-347); National Natural Science Foundation of China (No.51975567).

\section{Reference}

1. Thirsk R, Kuipers A, Mukai C, et al., Canadian Medical Association Journal, 180,12161220(2009).

2. Grosveld F W, Goodman J R, Pilkinton G D. Noise-Con 2003, the 2003 National Conference on Noise Control Engineering. 23-25(2003).

3. O'keefe E, Counter D. Space Programs and Technologies Conference and Exhibit. 4080(1993).

4. ZHONG Zuoyang, ZHANG Hailian, ZHOU Jianping. Manned Spaceflight. 6(2017)

5. Ma Xingrui Han Zengyao, Mechanical environment analysis method and test technology of satellite and launch vehicle.(2014). 
6. Larbi W, Deue J F, Ohayon R, et al. Coupled FEM/BEM for control of noise radiation and sound transmission using piezoelectric shunt damping[J]. Applied Acoustics, 2014, 86(dec.):146-153.

7. Citarella R, Federico L, Cicatiello A . Modal acoustic transfer vector approach in a FEM-BEM vibro-acoustic analysis[J]. Engineering Analysis with Boundary Elements, 2007, 31(3):248-258. 